\title{
Medical Toxicology and Public Health- Update on Research and Activities at the Centers for Disease Control and Prevention, and the Agency for Toxic Substances and Disease Registry
}

\section{Inhalational Methanol Toxicity}

\author{
Richard Kleiman, $M D^{a}$, Richard Nickle, $M P H^{b}$, Michael Schwartz, $M D^{a}$
}

aOffice of Terrorism Preparedness and Emergency Response (OTPER), National Center for Environmental Health (NCEH), Office of the Director

bDivision of Toxicology and Environmental Medicine, Agency for Toxic Substances and Disease Registry (ATSDR)

The following is an update on research and activities in which clinical toxicologists are actively involved at the National Center for Environmental Health and the Agency for Toxic Substances and Disease Registry (NCEH/ATSDR) at the Centers for Disease Control and Prevention (CDC). The Journal of Medical Toxicology periodically will highlight some of these activities to illustrate the growing relationship between clinical toxicology and public health.

\section{NCEH/ATSDR OFFICE OF TERRORISM PREPAREDNESS AND EMERGENCY RESPONSE}

The Office of Terrorism Preparedness and Emergency Response (OTPER) serves both the National Center for Environmental Health in the Centers for Disease Control and Prevention (CDC) and the Agency for Toxic Substances and Disease Registry (ATSDR). OTPER is CDC/ATSDR's primary point of contact for questions, requests for assistance, and access to subject matter expertise, laboratory resources, and emergency response technical support during incidents involving human exposure(s) to environmental agents and other public health consequences of nat- ural and manmade and/or technological disasters. An OTPER Duty Officer is available 24/7 to triage calls for assistance to specific OPDIVs (operating divisions) within CDC. In addition, the ATSDR Division of Toxicology and Environmental Medicine's duty officer is available to provide emergency response guidance, which may include technical assistance concerning chemicalspecific safety, environmental monitoring, and health guidance values, as well as advice on medical management-including personal protection, decontamination, diagnostics, and clinical care. The incident described below is an example of coordination between CDC and its partners in a successful response to an offshore incident with potential public health implications.

On May 24, 2008, CDC received a call requesting technical assistance regarding two men with a potential infectious disease or toxic exposure. The 2 crewmen worked on a tanker that carried chemicals, including methanol. The 2 affected individuals were tasked with cleaning a chemical storage tank after the methanol had been offloaded at the last port. One of the $2 \mathrm{crew}$ men died on board the vessel prior to its arrival in port, while the second was evacuated to a hospital. Both crewmen were trained and reportedly experienced in using personal protective equipment (PPE), and they were wearing appropriate protective equip-

Editor's Note: The findings and conclusions in this article are those of the authors and do not necessarily represent the views of the Centers for Disease Control and Prevention or the Agency for Toxic Substances and Disease Registry.

Acknowledgment: We would like to thank John Osterloh, M.D., for the considerable time and guidance he contributed to this publication. 
ment, including respirators, while cleaning the tank. The deceased crewman had reportedly developed a headache one night after cleaning the tank and was subsequently found the next morning unresponsive in his bunk with blood in his mouth. He was pronounced dead on board and placed in quarantine until the cause of death could be determined. Later autopsy ultimately revealed toxic levels of methanol and formic acid; the final cause of death was determined to be acute toxicity due to methanol poisoning. Of 7 potentially involved crewmen, 5 appeared to be unaffected and were asymptomatic, but they were originally thought to have the same potential exposure.

The second crewman became critically ill on the morning that the first was found dead, and was flown to a local hospital. On arrival at the hospital, he was noted to have a severe metabolic acidosis with a $\mathrm{pH}$ of 6.7 and an elevated anion gap of 31 . The calculated osmolality was $290 \mathrm{mOsmol} / \mathrm{kg}$, and the serum osmolality was $335 \mathrm{mOsmol} / \mathrm{kg}$, producing an osmol gap of 45 . However, the serum osmolality was drawn 7 hours after the chemistry panel used to determine the calculated osmolality, suggesting that the osmol gap could have been initially higher if it had been drawn at the same time. The patient was intubated and received bicarbonate therapy and aggressive supportive care. A complete work-up for toxic and infectious etiologies was conducted, and empirical treatment with fomepizole was initiated after the history suggested exposure to methanol. The blood methanol level was determined to be $89 \mathrm{mg} / \mathrm{dL}$. This patient's condition gradually improved, but upon extubation he was found to have suffered significant vision loss. He was discharged to a rehabilitation center.

\section{COMMENTARY}

Currently, there is an ongoing law enforcement and regulatory investigation regarding this event. While the autopsy on the first crewman and the laboratory data on the second patient suggest methanol toxicity as the etiology of their illnesses, several uncertainties remain. It is unknown whether the exposure was due to inhalational exposure, dermal exposure, or ingestion. While the route of exposure may be discovered during the investigation, this event provides an opportunity to discuss methanol toxicity, with a particular focus on occupational exposure, including inhalational exposure.

\section{INHALATIONAL METHANOL TOXICITY}

\section{Background}

Methanol is considered to be one of the toxic alcohols, a group that includes ethylene glycol and isopropyl alcohol. Methanol, also known as methyl alcohol, is a hydrocarbon with a hydroxyl group attached to its single carbon. It was initially distilled from wood, earning its common name "wood alcohol." It is still referred to as such today, but most methanol is now generated synthetically. It is a clear, colorless liquid at room temperature, and it has a slight odor. It was used historically as a component of embalming fluid
[1-2], and it is now used commonly as a solvent and as a component of different types of gasoline. It also is used in carburetor cleaners, and the compounds have been abused by people inhaling them to seek a "high" [3-6]. Methanol is used in the semiconductor industry [7] and by biotechnologists who work with liquid chromatography [8]. It is commonly used in varnishes, windshield wiper fluid, adhesives, and antifreeze [7]. Low-level exposure to methanol occurs from ingestion of fresh fruit and vegetables, the artificial sweetener aspartame when it is metabolized [9], and through endogenous demethylation metabolic pathways.

\section{Prevalence/Exposure}

Methanol toxicity in the United States occurs via ingestion of such compounds as windshield washer fluid, antifreeze, and cooking fuel such as Sterno. Outbreaks of methanol toxicity have also occurred in the United States and abroad as a result of ingestion of tainted alcohol products, such as moonshine [1,10-11]. There are cases of inhalational and dermal methanol toxicity reported in the literature, but these are less common and are often related to occupational exposure and abuse $[3-6,12]$. There are also reports of children who have died from dermal exposure to methanol [13]. Chronic and acute exposures to methanol vapors have been studied at or near the limits of allowable methanol vapor exposure $[9,14-15]$. The current occupational limits are 200 ppm in air averaged over 8-or 10-hr days and 40-hr weeks (Occupational Safety and Health Administration [OSHA] permissible exposure level [PEL], American Conference of Governmental Industrial Hygienists [ACGIH] threshold limit value [TLV], and National Institute of Occupational Safety and Health [NIOSH] recommended exposure limit [REL]), when measurements of $250 \mathrm{ppm}$ were averaged over 15 minutes (short-term exposure limit [STEL]). The Immediately Dangerous to Life and Health (IDLH) concentration established by the NIOSH is 6000 ppm [16].

\section{Pharmacokinetics}

Methanol is absorbed rapidly following ingestion and inhalation, but it has a slightly slower absorption following dermal exposure. Methanol levels after inhalational exposure may be related not only to the amount and time of exposure, but also to the effort and workload of the individual exposed. Therefore, someone with a higher workload in a certain environment for a set period of time might suffer a higher methanol uptake than someone in the same environment who is less active [15]. One study examining human experimental exposure to methanol found that the inhalational absorption half-life was 0.8 hours when volunteers were exposed to methanol vapors at TLV of $200 \mathrm{ppm}$ for 4 hours [14]. Once absorbed, methanol appears to be rapidly and fairly well distributed, with a volume of distribution of $0.7 \mathrm{l} / \mathrm{kg}$, consistent with its hydrophilicity [17-19]. Methanol has also been reported to cross the placenta and result in neonatal toxicity [20].

Similar to intoxication by ethanol and isopropanol, methanol intoxication results in early central nervous system (CNS) depression. However, its further metabolism will result in distinctive toxicity. Metabolism of methanol occurs primarily in the liver, 


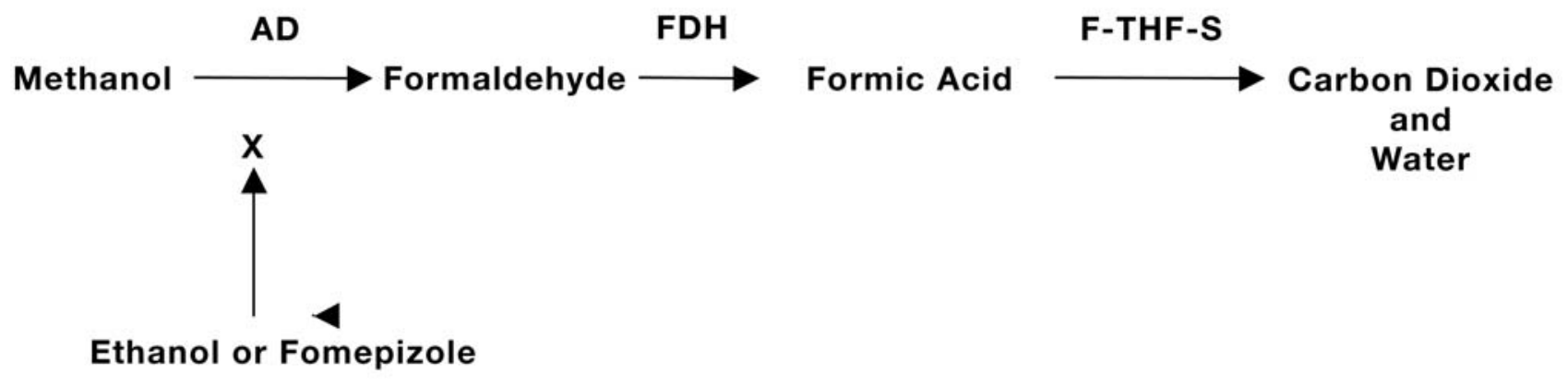

Figure 1: Methanol metabolism [26]. ADH, alcohol dehydrogenase; FDH, formaldehyde dehydrogenase; F-THF-S, 10-formyl tetrahydrofolate synthetase.

where alcohol dehydrogenase (ADH) oxidizes methanol to formaldehyde, which is subsequently converted to formic acid via formaldehyde dehydrogenase. This reaction occurs very rapidly, so that there is very little accumulation of formaldehyde in the bloodstream. The formic acid, in the presence of tetrahydrofolate, is then further metabolized to carbon dioxide and water by the enzyme 10-formyl tetrahydrofolate synthetase (Figure 1). Tetrahydrofolate is derived from folic acid, and when the system becomes overwhelmed, a buildup of formic acid develops and results in toxicity [21-25].

The elimination of high levels of methanol following large ingestions is a zero-order elimination process, but methanol follows first-order elimination at lower levels [26-30]. As stated previously, the NIOSH permissible exposure limit for inhalational methanol is $200 \mathrm{ppm}$, and studies have found an average elimination half-life of 3.2 hours when volunteers were exposed to this level [14], consistent with first-order elimination that is seen at low levels of ingestion. Similar first-order elimination was also found in individuals who were exposed to higher concentrations for a shorter period of time [31]. When treatment for methanol toxicity is initiated, the half-life of methanol can be as long as 54 hours [22]. At low levels, formate has a half-life of 1-3 hours, but the half-life can be prolonged at higher levels, likely due to saturation of elimination pathways or continued production [32]. Formate and methanol levels are good markers for occupational exposure [33-34]. Much of the data for inhalational and dermal exposure to methanol come from literature involving chronic exposure or case reports, and the known toxicokinetics may be altered in the case of a massive acute exposure.

\section{Pathophysiology}

The pathophysiology of methanol toxicity is closely tied to its metabolism to formic acid. As methanol is metabolized to formic acid, it creates the initial acidosis associated with methanol toxicity. The formate anion then inhibits the mitochondrial cytochrome oxidase, leading to lactate production. This is a self-inducing system in which as the acidosis worsens, more formic acid and lactate are produced. The acidosis also allows more formic acid to pass into the CNS, and it decreases the elimination of formic acid [22,35-36].

Formate causes specific injury to the optic nerve, and the worst injury appears to occur at the myelin sheath in the retrolaminar region. The optic nerve fibers may be more susceptible, secondary to lower levels of cytochrome oxidase [9,37]. In serious cases, injury may occur in the basal ganglia, with the putamen appearing to be very susceptible [38-40].

The pathophysiology of inhalational methanol toxicity appears to be similar to the better-characterized toxicity following ingestion. However, systemic bioavailability may be lower with chronic inhalational exposure [41]. As stated earlier, the overall toxicity appears to be dependent on the duration and air concentration, as well as on the workload of the person exposed. Neurological deficits have been seen with acute exposures to low levels of inhaled methanol, and it seems reasonable that higher levels of acute inhalational exposure could overwhelm the folatedependent pathway, resulting in greater toxicity.

\section{Clinical Presentation/Diagnosis}

The initial presentation of methanol intoxication may resemble other alcohol intoxication presentations, with inebriation and CNS depression. Methanol toxicity can also produce gastrointestinal symptoms that are typical of other alcohols, such as nausea and vomiting. The initial presentation of mild CNS depression may be the only initial complaint, with acidosis and neurological symptoms occurring after a period of 6-24 hours, during which methanol has been metabolized to formic acid. This latent period can be even longer if ethanol is present to competitively inhibit the metabolism of methanol. The latent period also depends on the amount of methanol present, and a person's ethanol tolerance may affect the initial presentation. Someone with a high ethanol tolerance may show minimal clinical signs of alcohol intoxication after methanol or ethanol exposure. Therefore, a normal neurological picture does not rule out potential methanol toxicity $[1,12,26,35,40,42]$.

As methanol is metabolized and the acidosis worsens, the respiratory rate may increase to compensate for the acidosis. Visual 
disturbances, such as spotty or blurry vision or a complete loss of vision, appear to be the most specific symptoms associated with methanol toxicity [42]. The patient may also have CNS findings such as headache or dizziness. Further CNS findings, such as seizures and coma, may also be present in severe cases, and are thought to be due to cerebral edema [44]. Chronic exposure to methanol vapor has been associated with headaches, dizziness, blurred vision, and nausea. There have also been infrequent reports of parkinsonism related to delayed effects after methanol vapor exposure [15,45-46].

As stated previously, patients may present early with subtle signs of inebriation but no other findings. Methanol initially causes an elevated osmolar gap, and this gap may be an early indicator of toxic alcohol poisoning. The magnitude of the osmolar gap can vary among individuals, and while it may aid in the diagnosis of exposure to toxic alcohols, a normal osmolar gap does not exclude toxic alcohol exposure. As the methanol is metabolized into formic acid, the osmolar gap will decline and the anion gap acidosis will begin to develop. If the acidosis progresses unchecked, it can lead to further CNS involvement, hypotension, and multisystem involvement. Co-ingestion of ethanol can inhibit alcohol dehydrogenase and slow the metabolism of methanol to formaldehyde and formate, thus slowing the onset of the acidosis. Ethanol also contributes to the osmolar gap, thus leading to an overestimate of the methanol present if ethanol is not known to be present or is not measured separately $[20,35,40,42]$.

It is possible to obtain a methanol serum level, but this usually takes several hours at least and will likely not be useful in the initial management of the patient. A serum level is often useful in deciding if dialysis is needed for clearance of methanol alone. Generally, a patient with a peak methanol level of $<20 \mathrm{mg} / \mathrm{dL}$ will not be symptomatic, and someone with a level $>50 \mathrm{mg} / \mathrm{dL}$ is considered potentially seriously toxic. However, these levels must be interpreted within a framework that accounts for the time of ingestion, possible ethanol inhibition of $\mathrm{ADH}$, current acid-base status, and overall presentation [47]. Generally, CNS imaging studies are unnecessary, except in severe and prolonged methanol intoxication in which injury to the basal ganglia, specifically the putamen, and cerebral edema can occur [38-40,46].

\section{Management}

The first step in the management of a methanol-toxic patient remains the same as with any potential poisoned patient. The patient must be stabilized first, with immediate attention paid to the airway and hemodynamic status. Unless exposure is ongoing, there is little role for decontamination. This is particularly true of patients incurring only inhalational exposure. If there has been liquid contamination of the patient's clothing, the clothes should be removed to stop any ongoing exposure. Some sources recommend gastric lavage, but there is little evidence to support such treatment, and the absorption of methanol is so rapid that it is probably of little utility [48].

An initial consideration at presentation is whether to institute treatment to inhibit the metabolism of methanol to formic acid.
Either ethanol or fomepizole can be administered to inhibit alcohol dehydrogenase (see Figure 1). However, such a step will not have any effect on formic acid that has already been created. It will inhibit further production of formic acid from any remaining methanol, however. Indications for treatment with ADHD inhibitors include a methanol level of $>20 \mathrm{mg} / \mathrm{dL}$ or a high suspicion of methanol ingestion with an elevated osmolar gap or significant acidosis $[22,26]$. Fomepizole is easier to administer than ethanol. In addition, monitoring of ethanol levels is required for adequately maintaining a sufficient ethanol concentration in the serum of about $100 \mathrm{mg} / \mathrm{dL}$.

Bicarbonate therapy also can be useful if the serum $\mathrm{pH}$ is less than 7.3. Formic acid is more toxic than formate, and in an acidic environment, the equilibrium shifts toward the production of more formic acid. This increased formic acid production then leads to increased tissue penetration, with inhibition of the cytochrome oxidase and a subsequently more acidic environment. Correction of the acidosis has also been found to increase the elimination of formic acid [49]. Reports have shown that both severe acidosis and coma are poor prognostic indicators, and severely acidotic patients have been found to have more residual vision effects [43].

Indications for hemodialysis include severe metabolic acidosis, vision changes, lack of response to supportive care, inability to correct electrolytes, and renal impairment limiting excretion. A serum methanol level of $>50 \mathrm{mg} / \mathrm{dL}$ has also been used traditionally as an indication for dialysis, but there is evidence that patients with levels this high can be treated successfully with fomepizole alone [50-51]. Interestingly, while dialysis has been found to clear formic acid, the actual amount of formic acid that is cleared may not be clinically significant [36,43,52-53]. Dialysis periods of 4-12 hours may be required.

There may also be a role for folinic or folic acid in the treatment of methanol-poisoned patients. It is known that the breakdown of formic acid to carbon dioxide and water occurs through a folate-dependent pathway. Animal studies have shown that rodents have higher tetrahydrofolate concentrations in their liver, thus are more resistant to methanol poisoning. This protection was lost when the rodents were made to be folic acid-deficient [54-55]. On the basis of this data, folinic acid or folic acid is sometimes recommended as a supplemental treatment.

\section{Prevention of Inhalational Exposure}

Confined space entries, such as those entered by the patients in this case, tragically are a recurring source of injury, and they commonly occur on cargo ships. The U.S. Occupational Safety and Health Administration (OSHA) established procedures for conducting such entries in 29 CFR 1910.146 and specifically for shipyards at 29 CFR 1915.12 [56]. While this incident occurred offshore on an international vessel, many of these procedures are followed in the international community as well. In this case, evidence indicates that the cargo tank was checked for toxic gases and flammable hydrocarbons before the crew members entered the tank. The investigator for the incident shared verbally the 
results of a confined space entry permit, which reported that the tank was within acceptable limits for entry by the crew. Instrument readings showed a low level of total organic compounds, no combustible gases, and an oxygen level above 19.5\%.

OSHA defines a confined space as one large enough for a person to enter but not designed for continuous occupancy. By definition, the space also has limited access or entry points and/or may be configured in such a way that the person entering may become trapped. The requirements for entering such a space include the performance of air-monitoring before entry. The air-monitoring should include testing for sufficient oxygen, flammability, and toxic gases. In order to be considered hazardous, a confined space would have $<19.5 \%$ or $>25 \%$ oxygen; be at $>10 \%$ above the lower flammable limit; or have toxic gases present above the permissible exposure limit or similar occupational standard. The tests must be conducted in a specific order, because some meters require certain conditions to be present in order to function properly (e.g., many combustible gas indicators require that sufficient oxygen be present to support combustion in order to provide an accurate reading) [56].

There are limitations found with various meters. Most real-time instruments can detect only 1 chemical at a time, and detection of 1 chemical may be interfered with by other present chemicals. Usually, these meters are based on the principle of ionizing the gases present, although instruments vary with respect to the measurement principle. In this case report, the meters described as being used were a "toxic gas meter," a "hydrocarbon meter," and an oxygen meter. Toxic gas meter is a generic term sometimes used to refer to ionization detectors, and hydrocarbon meter is a generic term sometimes used to refer to combustible gas indicators (also known as explosimeters) (personal communication with Rich Nickle).

In responding to the inquiries from the authorities at the scene of the poisoning of the 2 workers, CDC/ATSDR searched the Internet for ionization detectors, referred to by the manufacturers as toxic gas meters. The bulk of these instruments have an ionization energy of 10.5 electron volts (ev), meaning that they may detect any compound with a first- or second-order ionization potential (IP) of 10.5 or less. According to the NIOSH Pocket Guide to Chemical Hazards [57], the IP for methyl alcohol is 10.84 ev, a level that would not be detected by the bulk of the meters on the market. The low levels of total VOCs reported on the confined space entry permit were in the range of typical impurities found in methanol, with ionization potentials below 10.5. Methanol is flammable, and it should be detectable by the combustible gas indicator; however, these are relatively insensitive meters with high detection limits. The concentration in the tank could be in the range of the NIOSH IDLH without being detected by the combustible gas indicator or without showing significant oxygen deficiency (personal communication with Rich Nickle). The confined space entry permit requirements by OSHA represent a significant preventative measure that has reduced the number of injuries due to this type of occupational exposure. However, the requirements must be applied with due regard to the limitations of the technology employed, and an ionization detector with the correct ionization energy must be used. It is important to note that until the investigation of the incident is complete, details about the air monitoring actually performed on the vessel cannot be confirmed.

\section{CONCLUSION}

Methanol toxicity is still a relatively common poisoning worldwide. Exposure typically occurs through ingestion, but there is potential for occupational inhalational exposures as well. People with potential exposure to methanol in any form need to wear the appropriate personal protective equipment, and they need to be familiar with proper detection methods. Medical professionals need to understand the metabolism of methanol and why knowledge of this metabolism is important for the diagnosis and management of this unique poison. Several methods of treatment exist for methanol toxicity. Through development of an understanding of the pathophysiology behind the toxin, the appropriate measures can be taken.

\section{REFERENCES}

1. Flomenbaum NE, Goldfrank LR, Hoffman RS, Howland MA, Lewin NA, Nelson LS. Goldfrank's Toxicologic Emergencies. 8th ed. New York: McGraw Hill; 2006.

2. de Ketttenis P. The historic and current use of glycol ethers: a picture of change. Toxicol Lett 2005;156:5-11.

3. Velez LI, Kulstad E, Shepherd G, et al. Inhalational methanol toxicity in pregnancy treated twice with fomepizole. Vet Hum Toxicol 2003;45:28-30.

4. McCormick MJ, Mogabgab E, Adams SL. Methanol poisoning as a result of inhalational solvent abuse. Ann Emerg Med 1990;19:639-642.

5. LoVecchio F, Sawyers B, Thole D, et al. Outcomes following abuse of methanol-containing carburetor cleaners. Hum Exp Toxicol 2004;23:473-475.

6. Bebarta VS, Heard K, Dart RC. Inhalational abuse of methanol products: elevated methanol and formate levels without vision loss. Am J Emerg Med 2006;24:725-728.

7. Gaffney S, Moody E, McKinley M, et al. Worker exposure to methanol vapors during cleaning of semiconductor wafers in a manufacturing setting. J Occup Environ Hyg 2008;5:313-324.

8. Sullivan JB, Krieger GR. Clinical Environmental Health and Toxic Exposures. 2nd ed. Philadelphia: Lippincott Williams and Wilkins; 2001.

9. Kavet R, Nauss KM. The toxicity of inhaled methanol vapors. Crit Rev Toxicol 1990;21:21-50.

10. Levy P, Hexdall A, Gordon P, Boeriu C, et al. Methanol contamination of Romanian home-distilled alcohol. J Toxicol/Clin Toxicol 2003;41:23-28.

11. Kane RL, Talbert W, Harlan J, et al. A methanol poisoning outbreak in Kentucky. A clinical epidemiologic study. Arch Environ Health 1968;17:119-129. 
12. Aufderheide TP, White SM, Brady WJ, et al. Inhalational and percutaneous methanol toxicity in two firefighters. Ann Emerg Med 1993;22:1916-1918.

13. Kahn A, Blum D. Methyl alcohol poisoning in an 8-monthold boy: an unusual route of intoxication. J Pediatr 1979;94: 841-843.

14. Osterloh JD, D'Alessandro A, Chuwers P, et al. Serum concentrations of methanol after inhalation at $200 \mathrm{ppm}$. J Occup Environ Med 1996;38:571-576.

15. Ernstgard L, Shibata E, Johanson G. Uptake and disposition of inhaled methanol vapor in humans. Toxicol Sci 2005;88:30-38.

16. Hazardous Substances Databank. Toxicology Data Network. National Library of Medicine. National Institutes of Health. 2006. Available from: http://toxnet.nlm.nih.gov.

17. Graw M, Haffner HT, Althaus L, et al. Invasion and distribution of methanol. Arch Toxicol 2000;74:313-321.

18. Liesivuori J, Savolainen H. Methanol and formic acid toxicity: biochemical mechanisms. Pharmacol Toxicol 1991;69:157-163.

19. Jacobsen D, Jansen H, Wiik-Larsen E, et al. Studies on methanol poisoning. Acta Med Scand 1982;212:5-10.

20. Belson M, Morgan BW. Methanol toxicity in a newborn. J Toxicol/Clin Toxicol 2004;42:673-677.

21. Batterman SA, Franzblau A, D'Arcy JB, et al. Breath, urine, and blood measurements as biological exposure indices of short-term inhalation exposure to methanol. Int Arch Occup Environ Health 1998;71:325-335.

22. Brent J, McMartin K, Phillips S, et al. Fomepizole for the treatment of methanol poisoning. N Engl J Med 2001;344:424-429.

23. McMartin KE, Martin-Amat G, Noker PE, et al. Lack of a role for formaldehyde in methanol poisoning in the monkey.

Biochem Pharmacol 1979;28:645-649.

24. McMartin KE, Ambre JJ, Tephly TR. Methanol poisoning in human subjects. Role for formic acid accumulation in the metabolic acidosis. Am J Med 1980;68:414-8.

25. Eells JT, McMartin KE, Black K, et al. Formaldehyde poisoning. Rapid metabolism to formic acid. JAMA 1981;246: 1237-1238.

26. Barceloux DG, Bond GR, Krenzelok EP, et al. American Academy of Clinical Toxicology practice guidelines on the treatment of methanol poisoning. J Toxicol/Clin Toxicol 2002;40:415-446.

27. Eells JT, Black KA, Tedford CE, et al. Methanol toxicity in the monkey: effects of nitrous oxide and methionine. J Pharmacol Exp Ther 1983;227:349-353.

28. Jacobsen D, Webb R, Collins TD, et al. Methanol and formate kinetics in late diagnosed methanol intoxication. Med Toxicol Adverse Drug Exp 1988;3:418-423.

29. Wu AH, Kelly T, McKay C, et al. Definitive identification of an exceptionally high methanol concentration in an intoxication of a surviving infant: methanol metabolism by first-order elimination kinetics. J Forensic Sci 1995;40: 315-320.
30. Palatnick W, Redman LW, Sitar DS, et al. Methanol halflife during ethanol administration: implications for management of methanol poisoning. Ann Emerg Med 1995;26:202-207.

31. Perkins RA, Ward KW, Pollack GM. A pharmacokinetic model of inhaled methanol in humans and comparison to methanol disposition in mice and rats. Environ Health Perspect 1995; 103:726-733.

32. Osterloh JD, Pond SM, Grady S, et al. Serum formate concentrations in methanol intoxication as a criterion for hemodialysis. Ann Intern Med 1986;104:200-3.

33. Nihlen A, Droz PO. Toxicokinetic modelling of methyl formate exposure and implications for biological monitoring. Int Arch Occup Environ Health 2000;73:479-487.

34. Wahl A, Azaroual N, Imbenotte M, et al. Poisoning with methanol and ethylene glycol:1H NMR spectroscopy as an effective clinical tool for diagnosis and quantification. Toxicology 1998;128:73-81.

35. Hovda KE, Hunderi OH, Rudberg N, et al. Anion and osmolal gaps in the diagnosis of methanol poisoning: clinical study in 28 patients. Intensive Care Med 2004;30:1842-1846.

36. Kerns W 2nd, Tomaszewski C, McMartin K, et al. Formate kinetics in methanol poisoning. J Toxicol/Clin Toxicol 2002;40: 137-143.

37. Sharpe JA, Hostovsky M, Bilbao JM, et al. Methanol optic neuropathy: a histopathological study. Neurology 1982;32: 1093-1100.

38. Glazer M, Dross P. Necrosis of the putamen caused by methanol intoxication: MR findings. Am J Roentgenol 1993;160: 1105-1106.

39. Sefidbakht S, Rasekhi AR, Kamali K, et al. Methanol poisoning: acute MR and CT findings in nine patients. Neuroradiology 2007;49:427-435.

40. Fontenot AP, Pelak VS. Development of neurologic symptoms in a 26-year-old woman following recovery from methanol intoxication. Chest 2002;122:1436-1439.

41. Sedivec V, Mraz M, Flek J. Biological monitoring of persons exposed to methanol vapours. Int Arch Occup Environ Health 1981;48:257-271.

42. Frenia ML, Schauben JL. Methanol inhalation toxicity. Ann Emerg Med 1993;22:1919-1923.

43. Liu JJ, Daya MR, Carrasquillo O, et al. Prognostic factors in patients with methanol poisoning. J Toxicol/Clin Toxicol 1998;36:175-181.

44. Scrimgeour EM. Outbreak of methanol and isopropanol poisoning in New Britain, Papua New Guinea. Med J Aust 1980;2:36-38.

45. Finkelstein Y, Vardi J. Progressive parkinsonism in a young experimental physicist following long-term exposure to methanol. Neurotoxicology 2002;23:521-525.

46. Comoglu S, Ozen B, Ozbakir S. Methanol intoxication with bilateral basal ganglia infarct. Australas Radiol 2001;45:357-358.

47. Swartz RD, Millman RP, Billi JE, et al. Epidemic methanol poisoning: clinical and biochemical analysis of a recent episode. Medicine (Baltimore) 1981;60:373-382. 
48. Kruse JA. Methanol poisoning. Intensive Care Med 1992;18:391-397.

49. Hovda KE, Andersson KS, Urdal P, et al. Methanol and formate kinetics during treatment with fomepizole. Clin Toxicol (Phila) 2005;43:221-227.

50. Megarbane B, Borron SW, Trout H, et al. Treatment of acute methanol poisoning with fomepizole. Intensive Care Med 2001;27:1370-1378.

51. Hovda KE, Jacobsen D. Expert opinion: fomepizole may ameliorate the need for hemodialysis in methanol poisoning. Hum Exp Toxicol 2008;27:539-546.

52. Hantson P, Haufroid V, Wallemacq P. Formate kinetics in methanol poisoning. Hum Exp Toxicol 2005;24:55-59.

53. Hovda KE, Hunderi OH, Tafjord AB, et al. Methanol outbreak in Norway 2002-2004: epidemiology, clinical features and prognostic signs. J Intern Med 2005;258:181-190.
54. McMartin KE, Makar AB, Martin G, et al. Methanol poisoning. I. The role of formic acid in the development of metabolic acidosis in the monkey and the reversal by 4-methylpyrazole. Biochem Med 1975;13:319-333.

55. Lee EW, Garner CD, Terzo TS. Animal model for the study of methanol toxicity: comparison of folate-reduced rat responses with published monkey data. J Toxicol Environ Health 1994;41:71-82.

56. Permit-Required Confined Space Entry. Title 29, Code of Federal Regulations, part 1910.146. 1993. Available from: http://www.access.gpo.gov/nara/cfr/waisidx_08/29cfrv5_ 08.html.

57. Methyl Alcohol. NIOSH Pocket Guide to Chemical Hazards. National Institute for Occupational Safety and Health. Centers for Disease Control and Prevention. 2005. Available from: http://www.cdc.gov/niosh/npg/npgd0397.html. 